\title{
Early Detection of Fungal Infection in an Immunosuppressed Patient Demonstrated by Thorax CT: Suspicion is the Best Marker!
}

\author{
Gamze Durhan'1, Yusuf Baytar¹, Orhan Macit Arıyürek¹ \\ 1 Department of Radiology, Hacettepe University School of Medicine, Ankara, Turkey
}

2 6-year-old man with acute lymphoblastic leukemia developed neutropenic fever 12 days after induction chemotherapy. The focus of infection could not be found by clinical examination, chest radiography and routine cultures and empiric antimicrobial treatment was started. As the fever persisted, computerized tomography (CT) of the thorax was performed, and a nodule with a diameter of $2 \mathrm{~mm}$ in the upper lobe of the right lung was detected (Fig 1 \& 2). Absence of the lesion in the previous CT led us to think about a possible focus of infection. A follow-up CT taken one week later revealed that the size of nodule increased rapidly and reached a diameter of $13 \mathrm{~mm}$ (Fig 3). Fungal infection, especially angioinvasive aspergillosis, was considered in the differential diagnosis. A galactomannan index level of 2.0 in the bronchoalveolar lavage fluid further supported the radiological diagnosis. After antifungal treatment, including liposomal amphotericin B, the focus of infection regressed (Fig 4). The patient was discharged with the regression of fever and neutropenia.

The findings of chest radiographs are seldom specific for the detection of a particular pathogen in the patients with febrile neutropenia. Computed tomography of the thorax, especially high resolution and thin section, is more sensitive than chest radiograph and could enable detection of pneumonia about 5 days earlier than chest radiograph. On the other hand, CT demonstrates some specific findings in certain infections, narrows the list of potential pathogens and helps the clinician for the appropriate treatment (1-2).

Viral infections usually present with small poorly defined centrilobular nodules and consolidation foci and/or ground glass attenuation with peribronchial distribution, while bacterial types of pneumonia usually present with consolidation which may be focal, patchy or diffuse. Also, the findings in viral infections are multifocal and often bilateral (3). Conversely, the halo sign in a febrile patient with neutropenia is highly suggestive of angioinvasive aspergillosis infection. The halo sign is the CT finding of a peripheral rim of ground-glass opacity surrounding a pulmonary nodule or mass. It is an early sign which is seen only in the first 10 days of angioinvasion. These lesions progressively coalesce with

Corresponding Author: Gamze Durhan

E-mail: gamzedurhan@gmail.com

Received: July 27, 2019

Accepted: August 22, 2019 Published: October 7, 2019

Suggested citation: Durhan G, Baytar Y, Arıyürek OM. Early Detection of Fungal Infection in an Immunosuppressed Patient Demonstrated by Thorax CT: Suspicion is the Best Marker! Infect Dis Clin Microbiol 2019; 2: 107-109.

DOI: 10.36519/idcm.2019.19016 


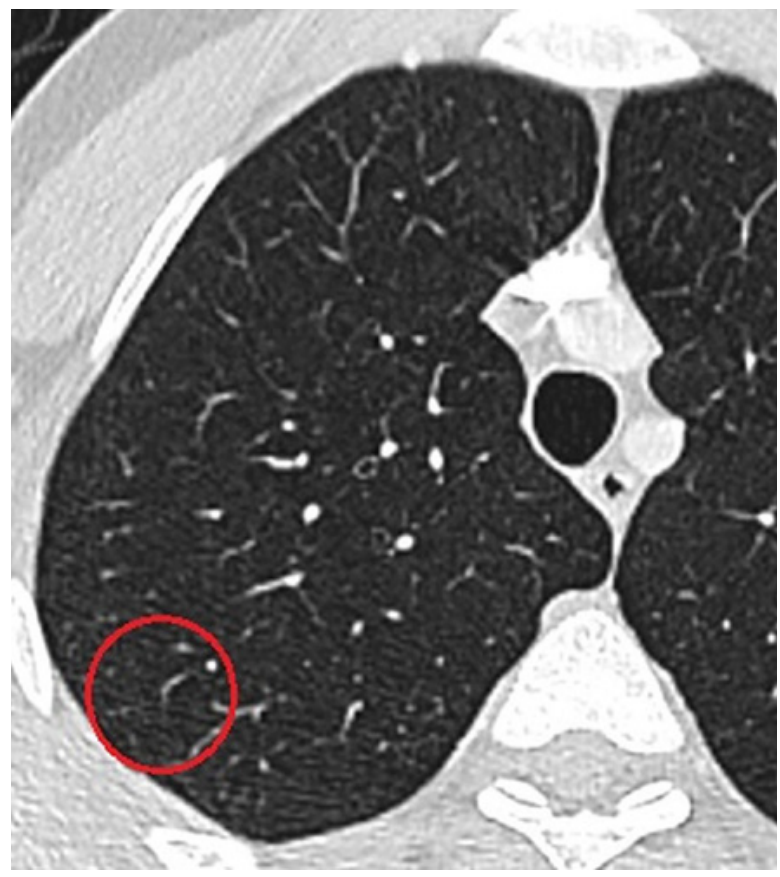

Figure 1. There was not any nodule in previous axial CT image of the patient.

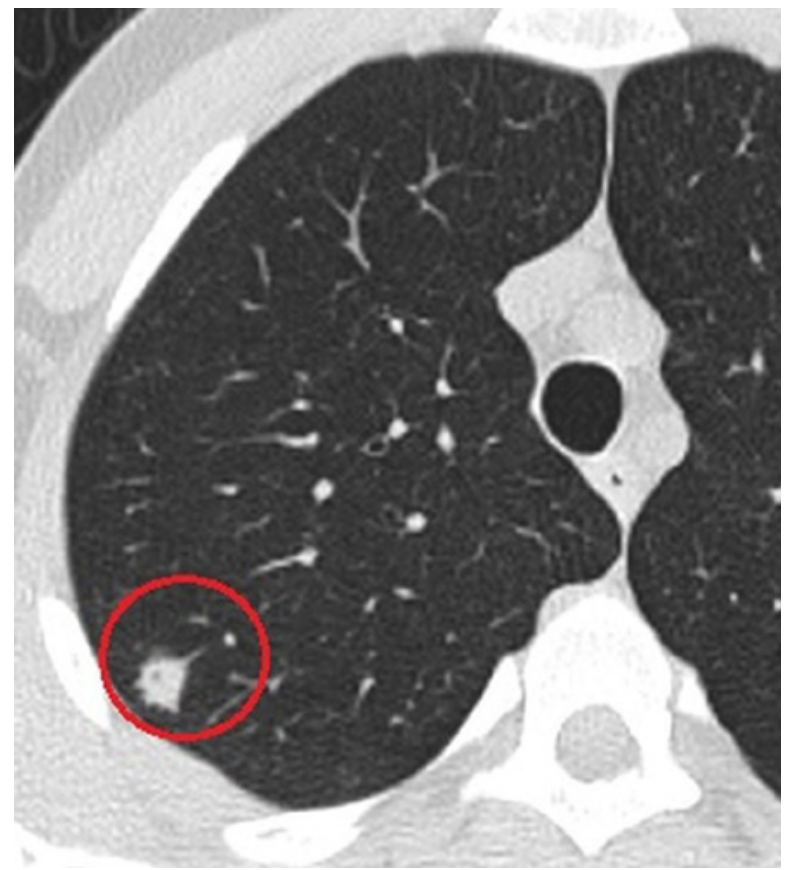

Figure 3. Follow-up CT scan which was obtained after 1 week, demonstrates the growing nodule with the halo sign suggesting the angioinvasive aspergillosis.

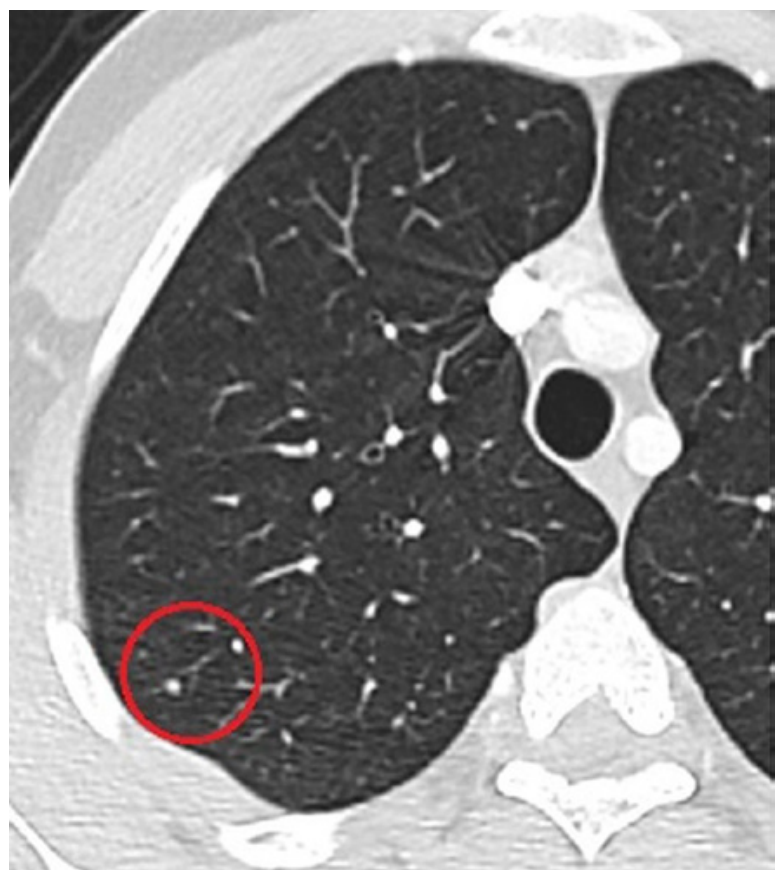

Figure 2. The axial CT image which was obtained when the patient had febrile neutropenia shows a new small nodule.

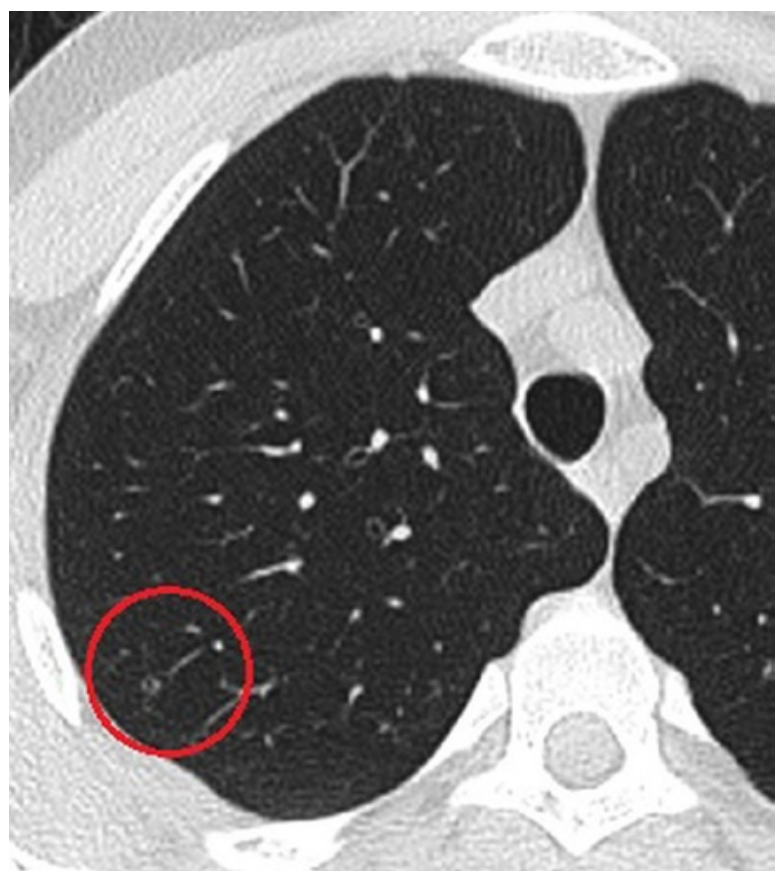

Figure 4. CT scan obtained after antifungal therapy shows the regression of the nodule. 
confluent consolidation (4). Early detection of this sign by $\mathrm{CT}$ provides appropriate therapy on time.

Predicting of angioinvasive aspergillosis in the presence of nodule with the halo sign may be partially easy. On the other hand, the nodules without halo sign can also be seen in angioinvasive aspergillosis (5). If a new nodule is detected in a patient with febrile neutropenia, fungal infection should be suspected regardless of the size of nodule.
Peer-review: Externally peer-reviewed.

Author Contributions: Concept - G.D., Y.B; Design - O.M.A.; Supervision - O.M.A.; Data Collection and/or Processing - G.D., Y.B; Analysis and/or Interpretation - G.D., O.M.A.; Literature Search - G.D., O.M.A.; Writing Manuscript - G.D., Y.B; Critical Review - Y.B, O.M.A.
Conflict of Interest: The authors have no conflict of interest to declare.

Financial Disclosure: The authors declared that this study has received no financial support.

\section{REFERENCES}

1 Oh YW, Effmann EL, Godwin JD. Pulmonary infections in immunocompromised hosts: the importance of correlating the conventional radiologic appearance with the clinical setting. Radiology 2000; 217: 647-56.

2 Heussel CP, Kauczor HU, Heussel G, Fischer B, Mildenberger P, Thelen M. Early detection of pneumonia in febrile neutropenic patients: use of thin-section CT. AJR Am J Roentgenol 1997; 169: 1347-53.

3 Demirkazik FB, Akin A, Uzun O, Akpinar MG, Ariyurek MO. CT findings in immunocompromised patients with pulmonary infections. Diagn Interv Radiol 2008; 14: 75-82.

4 Prasad A, Agarwal K, Deepak D, Atwal SS. Pulmonary Aspergillosis: What CT can Offer Before it is too Late! J Clin Diagn Res 2016; 10: TE01-5.

5 Greene RE, Schlamm HT, Oestmann JW, Stark P, Durand C, Lortholary $\mathrm{O}$, et al. Imaging findings in acute invasive pulmonary aspergillosis: clinical significance of the halo sign. Clin Infect Dis 2007; 44: 373-9. 